\title{
Combined epidural anesthesia and ultrasound guided peripheral nerve block for wound revision in a patient with peripartum cardiomyopathy -A case report-
}

\author{
Tai Kyung Gong, and Seong Su Kim \\ Department of Anesthesiology and Pain Medicine, Gangneung Asan Hospital, College of Medicine, Ulsan University, Gangneung, Korea
}

Peripartum cardiomyopathy (PPCM) is a rare complication that occurs between the late stage of pregnancy and six months after delivery. PPCM presents as symptoms of left ventricular dysfunction and it can be fatal unless treated promptly. Furthermore, anesthesia and surgery in such patients is a large challenge to anesthesiologists. First and foremost, the maintenance of stable hemodynamics is a major concern. We report a case of combined lumbar epidural anesthesia and both ilioinguinal and iliohypogastric nerve block under ultrasound guided for a wound revision in a 37-year-old woman diagnosed with PPCM after an emergency cesarean section. (Korean J Anesthesiol 2010; 59: 353-358)

Key Words: Anesthesia, Epidural, Ilioinguinal and iliohypogastric nerve block, Peripartum cardiomyopathy, Ultrasonography, Ventricular dysfunction.

Peripartum cardiomyopathy (PPCM) is a form of idiopathic acute heart failure that develops in mothers without previously known heart diseases, and occurs without decisive causatives associated with pregnancy from late pregnancy to 6 months after delivery [1]. It was mentioned for the first time in the 19th century. In the 1930s, it was discussed by differentiating it from maternal heart failure that developed in association with ischemia, heart valve, endocrinal diseases, infections, etc. The incidence was reported to be one in $1,300-15,000$ cases. The risk factors are old age, multiple pregnancy history, multiparity pregnancy, African ethnicity, obesity, pregnancy-induced hypertension, preeclampsia, alcohol intake, breast feeding and family history. In particular, it has been reported primarily in African multiparous mothers older than 30 years [2]. PPCM can be diagnosed by the absence of other apparent etiologies inducing cardiomyopathy and acute dilated left ventricular failure symptoms, as well as by performing echocardiography.

Serious consequences can develop if paripartum cardiomyopathy patients are not diagnosed rapidly and treated appropriately. Anesthesia is also difficult and dangerous in such

Received: August 17, 2009. Revised: 1st, August 31, 2009; 2nd, September 21, 2009. Accepted: October 5, 2009.

Corresponding author: Seong Su Kim, M.D., Department of Anesthesiology and Pain Medicine, Gangneung Asan Hospital, College of Medicine, Ulsan University, 415, Bangdong-ri, Sacheon-myeon, Gangneung 210-711, Korea. Tel: 82-33-610-3402, Fax: 82-33-641-8180, E-mail: sskim@gnah.co.kr (c) This is an open-access article distributed under the terms of the Creative Commons Attribution Non-Commercial License (http:// creativecommons.org/licenses/by-nc/3.0/), which permits unrestricted non-commercial use, distribution, and reproduction in any medium, provided the original work is properly cited. 
patients. In a 37-year-old mother diagnosed with preeclampsia, left ventricular dysfunction symptoms were noted during general anesthesia for an emergency Cesarean section. She was diagnosed with PPCM by echocardiography. In patients under treatment for PPCM, epidural anesthesia and a nerve block are performed in parallel for wound revision generally with a successful outcome. We report this case along with a review of the relevant literature.

\section{Case Report}

A 37-year-old parous woman, 36 weeks and 4 days pregnancy, $52.5 \mathrm{~kg}$ in weight, and $153 \mathrm{~cm}$ in height with a pregnancy history of 1-0-0-1 was admitted for the chief complaints of labor. The mother had not been under prenatal care after 24 weeks of pregnancy for financial reasons. At the time of admission, her blood pressure, heart rate, respiratory rate and body temperature was $140 / 100 \mathrm{mmHg}, 80$ times/min, 20 times/ min, and $36.8^{\circ} \mathrm{C}$, respectively. Hypertension and proteinuria were detected, and she was diagnosed with preeclampsia. Accordingly, an emergency cesarean section was decided. The patient did not have a prior or family history, and did not consume alcohol or cigarettes. The physical examination revealed no special findings except for mild general edema, and no abnormal sounds were heard in chest ascultation. In the blood test, hemoglobin ( $\mathrm{Hb}$ ) was $6.9 \mathrm{~g} / \mathrm{dl}$, hematocrit (Hct) was $24.2 \%$, and blood coagulation, etc., were normal. A finding of albumin $3(+)$ was noted in the urine test. The chest radiographs revealed slight congestion of the pulmonary blood vessels, pulmonary edema findings and the Kerley B-line (Fig. 1). A

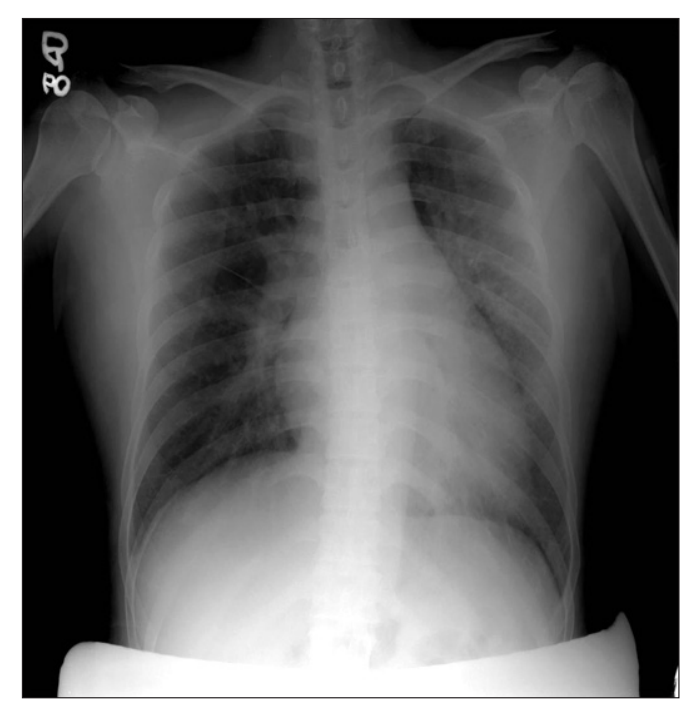

Fig. 1. Pre-operative chest PA shows mild pulmonary infiltration along the broncho-vascular bundle in both lung fields and Kerley B-line. normal sinus rhythm was observed on the electrocardiogram. No medication was administered before anesthesia.

Upon arriving at the operating room, an electrocardiography, noninvasive blood pressure monitor, and pulse oximeter were applied. Her vital signs at that time were as follows: blood pressure, heart rate, respiratory rate and body temperature of 148/112 mmHg, 123 times $/ \mathrm{min}, 20$ times $/ \mathrm{min}$ and $37.0^{\circ} \mathrm{C}$, respectively. Since severe labor pain and mild dyspnea were presented, it was decided to perform general anesthesia. For the induction of anesthesia, unconsciousness was induced by injecting $200 \mathrm{mg}$ thiopental sodium, followed by $40 \mathrm{mg}$ rocuronium (Esmeron ${ }^{\circledR}$, Hanwha Pharmaceuticals, Yongin, Korea). Manual ventilation was performed. To prevent the increase in blood pressure due to tracheal intubation, $5 \mathrm{mg}$ labetalol (Labesin ${ }^{\circledR}$, Myungmoon Pharmacueticals, Seoul, Korea) was administered, and tracheal intubation was performed 90 seconds after administering the muscle relaxant. One minute after intubation, her systemic blood pressure and heart rate was 120/95 $\mathrm{mmHg}$ and 95 times/min, respectively. General anesthesia was maintained with $\mathrm{O}_{2} 2 \mathrm{~L} / \mathrm{min}, \mathrm{N}_{2} \mathrm{O} 2 \mathrm{~L} /$ min, sevoflurane 1.2 vol\% under a Pfannenstiel skin incision. The Cesarean section was then initiated. Expulsion of the fetus occurred 8 minutes after the initiation of surgery. At that time, the 1 minute/5 minutes Apgar score was recorded as 6/9 points. After birth, 10 units oxytocin was added to the fluid and injected, and $100 \mu \mathrm{g}$ fentanyl was injected. Surgery proceeded smoothly. From 5 minutes after birth, at $\mathrm{FiO}_{2}$ 0.5, the oxygen saturation on the pulse oximeter became unstable as $90-$ 95\%. Accordingly, $\mathrm{N}_{2} \mathrm{O}$ administration was terminated, and arterial blood gas analysis was performed. In the arterial blood

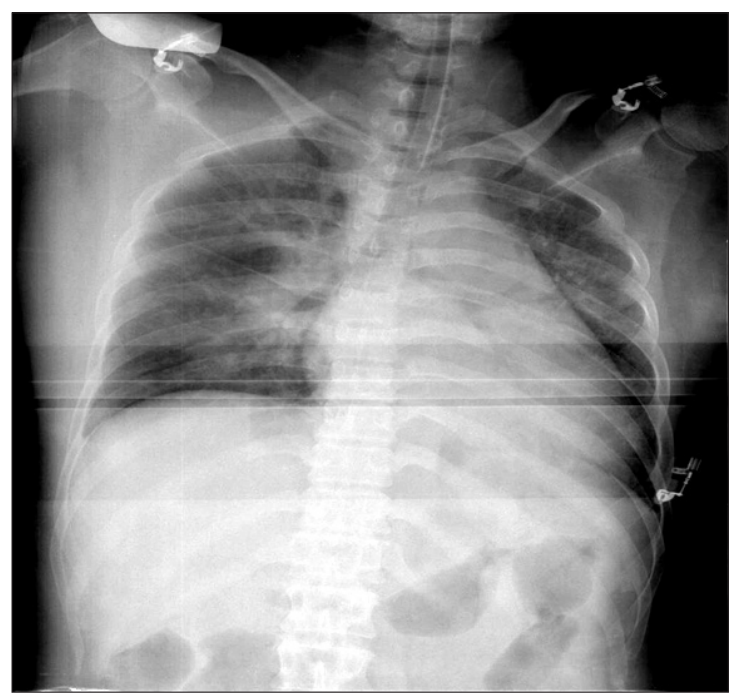

Fig. 2. Intra-operative chest AP shows more aggravated peri-vascular infiltration and prominent Kerley B-line. 
analysis, the $\mathrm{pH}, \mathrm{PaCO}_{2}, \mathrm{PaO}_{2}$, and $\mathrm{SaO}_{2}$ was $7.42,33 \mathrm{mmHg}$, $91 \mathrm{mmHg}$, and $96 \%$, respectively. In ascultation, pulmonary edema was suspected, and $5 \mathrm{mg}$ lasix was administered, and a chest X-ray was taken. In the chest X-ray, the pattern of infiltration to the vicinity of both pulmonary vascular bundles was observed, and bilateral Kerley B-line findings were noted (Fig. 2). Electrocardiography performed during surgery did not reveal any changes. After surgery, the self respiration of the patient began to recover, and $0.4 \mathrm{mg}$ glycopyrrolate and $15 \mathrm{mg}$ pyridostigmine were administered for the reversal of muscle relaxation. Subsequently, the patient recovered consciousness but pulse oximetry revealed an oxygen saturation of approximately $95 \%$. Considering the pattern of pulmonary edema on the chest X-ray performed during surgery, the patient in the state of endotracheal intubation was transported to the intensive care unit. The fluid administered during surgery, blood loss volume and total urine volume was 800 $\mathrm{ml}$ Hartmann's solution, approximately $500 \mathrm{ml}$ and $90 \mathrm{ml}$, respectively.

After transport to the intensive care unit, a mechanical ventilator was installed, and pressure controlled ventilation was performed at $\mathrm{FiO}_{2}$ 0.6, $10 \mathrm{mmHg}$ end tidal positive pressure, and $30 \mathrm{mmHg}$ maximal inspiratory pressure. Midazolam was administered continuously to maintain sedation. Echocardiography performed on day 1 after surgery revealed the left ventricular ejection fraction (LVEF) was $26 \%$, and the findings of dilated left ventricular failure, moderate mitral valve regurgitation, mild tricuspid valve regurgitation, and pulmonary arterial hypertension. Therefore, under the diagnosis of PPCM, the patient was transferred to the department of internal

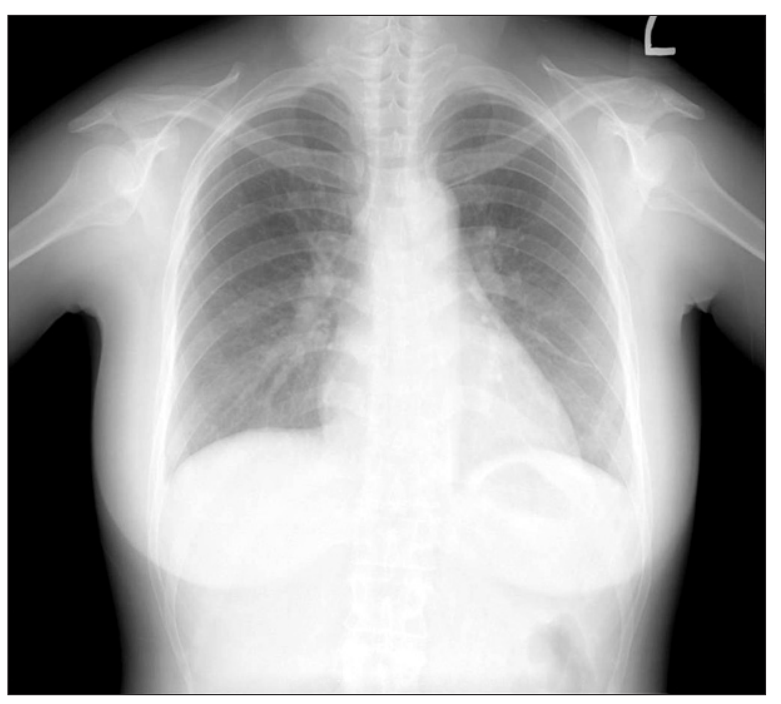

Fig. 3. Chest PA at post-operative day 4 shows a lower amount of both pleural effusion and improved sub-segmental collapse in the left lower lung field than the previous one. medicine. In the intensive care unit, the patient was under an absolute bed resting state, and lasix, aldacton, dexamethasone, hydralazine, dobutamine, and $\mathrm{MgSO}_{4}$ were administered. On day 2 after surgery, her symptoms improved, and disconnection of the mechanical ventilator and extubation of endotracheal intubation were performed. Subsequently, the patient was conscious with a stable blood pressure, heart rate and respiratory rate of 130/70 $\mathrm{mmHg}, 60$ times/min and 15 times/ min, respectively.

In the blood test, Hb, Hct, glutamic oxaloacetic transaminase/ glutamic pyruvic transaminase, blood urea nitrogen/creatinine, electrolyte and blood glucose levels were $9.1 \mathrm{~g} / \mathrm{dl}, 30.4 \%, 17 / 6$ $\mathrm{IU} / \mathrm{L}, 3.1 / 0.6 \mathrm{mg} / \mathrm{dl}, \mathrm{Na}^{+} 141 \mathrm{mEq} / \mathrm{L}, \mathrm{K}^{+} 3.3 \mathrm{mEq} / \mathrm{L}, \mathrm{Ca}^{2+} 7.6$ $\mathrm{mEq} / \mathrm{L}$, and $61 \mathrm{mg} / \mathrm{dL}$, respectively. Arterial blood gas analysis revealed a $\mathrm{pH}, \mathrm{PaCO}_{2}, \mathrm{PaO}_{2}$ and $\mathrm{SaO}_{2}$ of 7.67, $14 \mathrm{mmHg}, 128$ $\mathrm{mmHg}$ and $99 \%$, respectively. On day 4 after surgery, the vital signs of the patient were stable. On echocardiography, the LVEF was improved to $35 \%$. Compared to day 1 after surgery, reduced mitral valve regurgitation and tricuspid valve regurgitation were noted, and the patient was transferred to the general ward. Similarly, chest X-ray revealed apparent improvement in the features (Fig. 3).

However, from day 2 after surgery, continuous exudate in the incision wound of the patient was detected, and it was decided to perform additional surgery on day 8 after surgery to treat the incisional wound. The blood coagulation battery was normal and the area requiring a sensory block by a Pfannenstiel skin incision was not wide. It was decided to perform an ultrasound guided bilateral ilioinguinal nerve block and iliohypogastric nerve block together with epidural anesthesia to avoid myocardial suppression and rapid fluctuation of blood pressure caused by general anesthesia. Prior to the induction of anesthesia, a catheter was placed in the right radial artery for continuous monitoring of the arterial blood pressure and in the right subclavian vein to monitor the central venous blood pressure. An electrocardiogram, pulse oxygen saturation level, and the end of expiration carbon dioxide partial pressure $\left(\mathrm{EtCO}_{2}\right)$ were monitored. First, for epidural anesthesia, the patient was positioned in the left lateral position and the epidural space between the 2-3 lumbar vertebra was assessed with a 17-gauge Tuohy needle using a loss of resistance method. An epidural catheter was inserted and approximately $4 \mathrm{~cm}$ was placed in the epidural space. The position of the patient was changed to the supine position. A solution containing $8 \mathrm{ml} 2 \%$ lidocaine and $100 \mu \mathrm{g}$ fentanyl was injected through the epidural catheter. After 10 minutes, to perform bilateral ilioinguinal and iliohypogastric nerve blocks, the tip of a 5-10 MHz linear probe was placed in the anterior superior iliac spine, and twisted in the direction in parallel to the inguinal line. The external oblique muscle, the internal oblique muscle, and the 


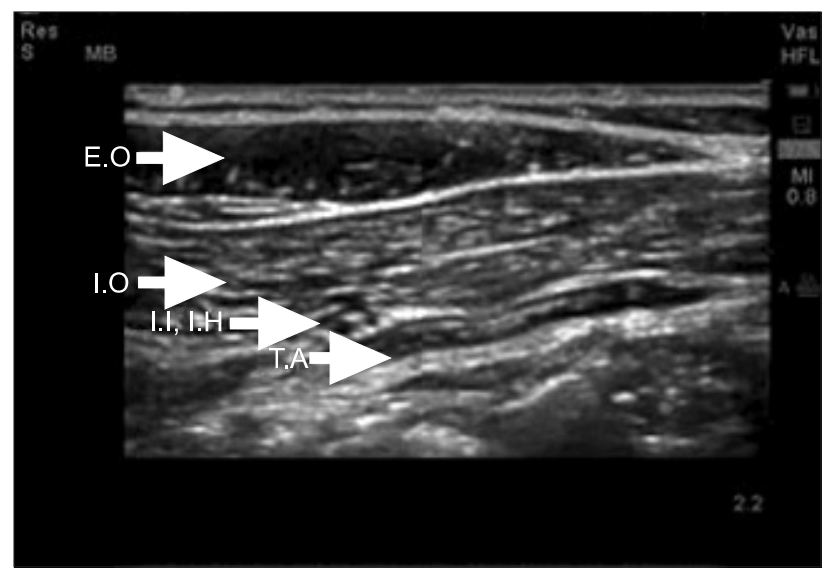

Fig. 4. Ultrasonographic images of the lower abdomen demonstrate the anatomy around ilioinguinaland iliohypogastric nerve. E.O: external oblique muscle, I.O: internal oblique muscle, T.A: transverse abdominis muscle, I.I: ilioinguinal nerve, I.H: iliohypogastric nerve.

transverse abdominus muscle were then assessed. A SonoSite 180plus portable ultrasound unit (SonoSiteTM ${ }^{\circledR}$, Bothell, WA, USA) was used for ultrasonography. Using a 22-gauge $40 \mathrm{~mm}$ needle, the tip of the needle in the optimal site in the vicinity of the nerve was assessed under ultrasonography guidance, and a bilateral ilioinguinal nerve block and iliohypogastric nerve block were performed by administering $20 \mathrm{ml} 1 \%$ lidocaine (Fig. 4 and 5). The accurate level of epidural block could not be assessed due to the uncooperative behavior of the patient, who reported pain in all areas. Nevertheless, pain was not reported while inserting the needle and it was determined that a sensory block in more than T12 was achieved. After injecting a total $10 \mathrm{ml}$ of the drug through the epidural catheter, the blood pressure and heart rate rarely changed, even though fluid was not supplied. Therefore, it was determined that a block more than required had not been induced. Surgery was performed smoothly without administering additional anesthetics and reporting pain, and the total operation time was 90 minutes. Blood loss barely occurred, and as fluid, $150 \mathrm{ml}$ of Hartmann's solution was injected, and the total urine volume was $30 \mathrm{ml}$. During surgery, the central venous pressure was maintained at 6-8 mmHg, and no special hemodynamic changes occurred. In echocardiography performed 15 days after the first Cesarean section, improved findings of $45 \%$ LVEF, and an improvement of the enlarged left ventricle, mild systolic impairment, and mitral valve regurgitation were shown. On the chest X-ray, clear improvement was noted. The vital signs showed normal findings: systemic blood pressure, heart rate, respiratory rate and body temperature of 120/60 $\mathrm{mmHg}, 70$ times/min, 15 times/min, and $36.5^{\circ} \mathrm{C}$, respectively. Therefore, on day 16 after surgery, without presenting with special symptoms, an ambulatory follow up observation was decided, and the patient

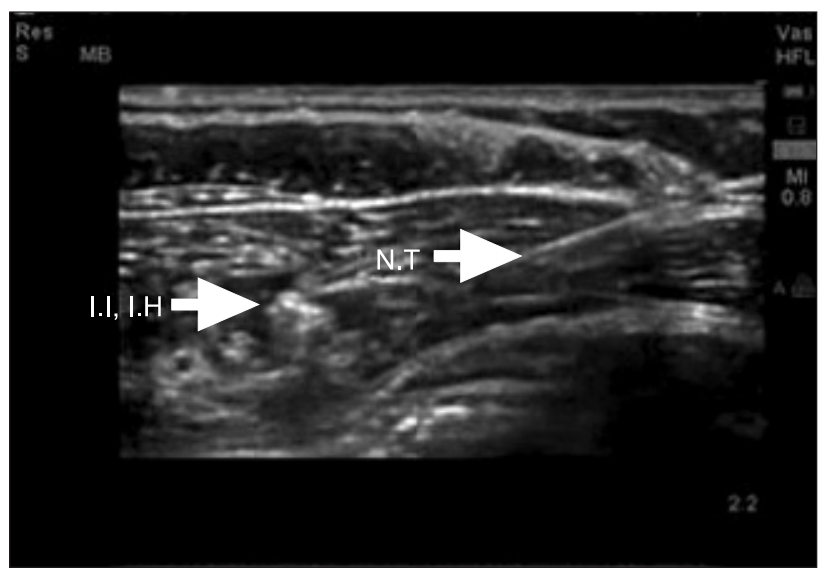

Fig. 5. Nerve block needle can be seen near the ilioinguinal and iliohypogastric nerves. I.I: ilioinguinal nerve, I.H: iliohypogastric nerve, N.T: needle tip.

was discharged.

\section{Discussion}

Since PPCM does not show a special history of heart disease or heart failure, most cases are detected incidentally during delivery or surgery. Nevertheless, our case was a PPCM patient diagnosed after a Cesarean section. The condition deteriorated requiring surgery for wound revision. It is difficult to plan the anesthesia of a patient who has not recovered completely from heart failure caused by PPCM. Moreover, a comprehensive understanding of PPCM as well as a complete assessment of the patient's condition will assist in the safe management of anesthesia.

Pregnancy-induced hypertension and preeclampsia have been reported to be closely associated with PPCM. The causes of paripartum cardiomyopathy has not been clearly established. Nevertheless, inflammatory myocarditis, cytokines, hemodynamic changes caused by pregnancy, hormonal changes, etc., have been suggested. From etiological aspects, an abnormal concentration of plasma releaxin, selenium deficiency, presence of pro-inflammatory cytokines, abnormal antibody reaction to heart tissue proteins, and inflammatory myocarditis, have been suggested. A rapid diagnosis of PPCM is reasonably difficult. PPCM presents as abnormal chest pain, dyspnea, orthopnea, coughing, fatigue and other left ventricular failure symptoms. Nevertheless, fatigue is a common symptom during the peripartum and late pregnancy period, and similarly, tachypnea is difficult to differentiate from other diseases that can induce tachypnea, such as normal pregnancy, pneumonia, asthma, pulmonary embolism, etc., making an early diagnosis is difficult. In electrocardiography, sinus tachycardia, atrial 
flutter and fibrillation may be shown, and diverse findings, such as left ventricular hypertrophy, etc., may be shown [3,4]. On chest X-ray, cardiomegaly, pulmonary edema, pleural effusion, and other findings may be noted. However, as in our case, almost normal findings may be shown. Echocardiography is the most important diagnostic method, and information on the severity of paripartum cardiomyopathy and its prognosis could be obtained [3]. Information on the diameter of the left ventricle at the end-diastolic phase, decreased LVEF $(<30 \%)$, changes to the heart wall, heart valve abnormality, epicardial effusion, etc., could be obtained. In addition, an endocardial biopsy or the insertion of cardiac catheter can provide more information for a diagnosis $[3,5,6]$. Concerning our patient, under the condition without a specific past history of heart disease prior to admission, the patient showed the following: dyspnea, pulmonary edema findings on the chest X-ray, $26 \%$ of the left ventricular ejection rate on echocardiography, dilated left ventricular failure findings, moderate mitral valve regurgitation, mild tricuspid valve regurgitation and pulmonary arterial hypertension findings. Therefore, she was diagnosed with cardiomyopathy. The treatments for PPCM are similar to heart failure, and include absolute bed rest, diuretics, vasodilators, angiotensin converting enzyme (ACE) inhibitors. In addition, anticoagulation treatment may be performed to prevent the formation of thrombus. Among them, ACE inhibitors are drugs that can induce a fetal deformity, and its use before delivery may be limited. Digoxin can also be used, and the administration of immune suppressors is still controversial. In addition, a heart transplant can be considered for cases unresponsive to all treatments [7].

For our patient, who suffered heart failure state due to PPCM, a wound revision was planned after a Cesarean section. General anesthesia and a combination of spinal anesthesia and epidural aensthesia etc. were introduced for the induction and maintenance of anesthesia for PPCM [8]. The advantages of general anesthesia are that it can avoid collapse of the cardiovascular system caused by the limited heart reservoir and collapse of cardiovascular system due to systemic vascular resistance that might occur during spinal anesthesia. In addition, invasive cardiovascular procedures, such as pulmonary capillary wedge pressure, central venous pressure, and transesophageal echocardiography, etc., can be performed during anti-coagulant treatments, and positive pressure ventilation through tracheal intubation is possible for cases associated with pulmonary edema $[9,10]$. On the other hand, the shortcomings of general anesthesia are that it can induce the severe suppression of the cardiopulmonary function of the mother and fetus. Moreover, it has been reported that serious bradycardia can even induce cardiac arrest [10]. During general anesthesia, the advantages of the use of opioids and the possibility of the consequent respiratory suppression of the fetus should be considered [11]. Anesthesiologists preferring regional anesthesia suggested that rapid effects could be manifested using a combination of epidural anesthesia and spinal anesthesia while avoiding the cardiovascular collapse phenomenon caused by the decrease in general vascural resistance. A satisfactory success rate of anesthesia can be obtained through the insertion of a catheter for epidural anesthesia, and hemodynamic instability caused by endotracheal intubation and volatile anesthetics can be avoided. In addition, an increase in cardiac output due to a decrease in cardiac afterload can be anticipated. Continuous pain control after surgery through the inserted epidural catheter is feasible, which can also better prevent thrombosis compared to general anesthesia, etc. [11]. Nevertheless, during regional anesthesia for PPCM patients, pulmonary arterial wedge pressure or the application of invasive monitoring, such as transesophageal echocardiography, cannot be performed readily on patients who are unstable hemodynamically or are currently under anti-coagulation therapy, and postdural puncture headache can develop after spinal tapping $[4,12]$. Regardless of the selection of anesthesia, the hemodynamic change in the patient should be monitored properly through pulmonary capillary wedge pressure, etc., when appropriate monitoring of central venous pressure, arterial pressure, and transesophageal echocardiography are required. In our patient, the dermatome was that wound revision of a pfannenstiel skin incision was limited, and epidural anesthesia and nerve block were performed in combination. The nerve block was performed under ultrasound guidance to reduce the dose of the local anesthetics administered, lower the failure rate of the procedure, and avoid the risk of the perforating the peritoneum during the procedure because the peritoneum was quite close to the target nerve [13]. Epidural anesthesia was performed in parallel because the dermatome range of the wound of the Pfannenstiel skin incision was not wide. Nevertheless, the accurate state could not be assessed before opening the wound. Furthermore, the possibility that a high anesthesia level equivalent to abdominal surgery would be needed cannot be ruled out if the surgery range is extended to the peritoneum due to traction of the peritoneum.

A nerve block can minimize the hemodynamic fluctuations better than epidural anesthesia, and the risk of the perforation of the peritoneum is not high with ultrasound guidance. Moreover, the purpose of a nerve block was major anesthesia and epidural anesthesia can be used to adjust the block level if needed. In contrast to expectation, the duration of actual surgery requiring revision of a part of the peritoneum was not short, and more anesthesia should be considered if an epidural catheter is not inserted. The patient presented with slight 
discomfort but the level of anesthesia was deemed sufficient by surgeons minimizing the traction of the peritoneum and anesthesiologists encouraging the patient. Surgery could be completed without an additional epidural injection of drugs. The combination of epidural anesthesia and spinal anesthesia as another method was not performed due to the concerns regarding the rapid hemodynamic fluctuations during spinal anesthesia. In regard to sedation after epidural anesthesia, sedation is difficult if the patient's pain is severe. Increasing the level of anesthesia by the additional administration of drugs itself deviates from the aim of anesthesia to minimize hemodynamic fluctuations, but was considered as another choice. Furthermore, the ilioinguinal and iliohypogastric nerves control the sensation of the L1 and L2 dermatome, and can be applied to inguinal hernia surgery, etc.

In conclusion, anesthesia for PPCM patients requires anesthetic procedures that can minimize the hemodynamic fluctuations and allow comprehensive monitoring of patients. Ultrasound guided nerve block performs the block while assessing the real time anatomical structure. It has advantages in that it reduces the dose of local anesthetics, lowers the failure rate of surgery, and avoids the risk of perforating the peritoneum. Among the anesthetic procedures for peripartum patients, epidural anesthesia and ultrasound-guided nerve block were performed in parallel, and surgery was completed without complications. This method can be a substitute for the anesthesia of PPCM patients.

\section{References}

1. Lampert MB, Lang RM. Peripartum cardiomyopathy. Am Heart J
1995; 130: 860-70

2. Witlin AG, Mabie WC, Sibai BM. Peripartum cardiomyopathy: an ominous diagnosis. Am J Obstet Gynecol 1997; 176(1 Pt 1): 182-8.

3. Heider AL, Kuller JA, Strauss RA, Wells SR. Peripartum cardiomyopathy: a review of the literature. Obstet Gynecol Surv 1999; 54: 526-31.

4. Ro A, Frishman WH. Peripartum cardiomyopathy. Cardiol Rev 2006; 14: 35-42.

5. Sanderson JE, Adesanya CO, Anjorin FI, Parry EH. Postpartum cardiac failure: heart failure due to volume overload? Am Heart J 1979; 97: 613-21.

6. Sliwa K, Fett J, Elkayam U. Peripartum cardiomyopathy. Lancet 2006; 368: 687-93.

7. Rickenbacher PR, Rizeq MN, Hunt SA, Billingham ME, Fowler MB. Long-term outcome after heart transplantation for peripartum cardiomyopathy. Am Heart J 1994; 127: 1318-23.

8. Lee HJ, Youn HJ, Chon JY, Choi YW, Moon SH. Anesthesia for cesarean section in a parturient with peripartum cardiomyopathy. Korean J Anesthesiol 2000; 39: 278-83.

9. Hutchinson RC, Ross AW. Severe peripartum cardiomyopathy. Anaesth Intensive Care 1992; 20: 398.

10. Malinow AM, Butterworth JF 4th, Johnson MD, Safon L, Rein M, Hartwell B, et al. Peripartum cardiomyopathy presenting at cesarean delivery. Anesthesiology 1985; 63: 545-7.

11. Kaufman I, Bondy R, Benjamin A. Peripartum cardiomyopathy and thromboembolism: anesthetic management and clinical course of an obese, diabetic patient. Can J Anaesth 2003; 50: 161-5.

12. Velickovic IA, Leicht CH. Peripartum cardiomyopathy and cesarean section: report of two cases and literature review. Arch Gynecol Obstet 2004; 270: 307-10.

13. Marhofer P, Greher M, Kapral S. Ultrasound guidance in regional anaesthesia. Br J Anaesth 2005; 94: 7-17. 